\title{
Robotic Exoskeleton: A Compact, Portable, and Constructing Using 3D Printer Technique for Wrist-Forearm Rehabilitation
}

\begin{abstract}
Authors affiliations:
1) Biomedical Engineering

Department, Al-Nahrain

University, Baghdad, Iraq, noorsabri93@yahoo.com.

$\left.2^{*}\right)$ Prosthetics and Orthotics Engineering Department, AlNahrain University, Baghdad, Iraq,

wajdisadik@gmail.com
\end{abstract}

\section{Paper History:}

Received: $28^{\text {th }}$ Feb. 2020

Revised: $21^{\text {st }}$ April 2020

Accepted: $16^{\text {th }}$ July 2020

\author{
Noor S. Shalal ${ }^{1}$, Wajdi S. Aboud ${ }^{2}$
}

\begin{abstract}
Regaining the activities of daily living after stroke and spinal cord injury requires repetitive and intensive tasks, meaning that rehabilitation therapy should be treated with a long duration. Thus, the need for rehabilitation devices based home is of most importance to increase the rehabilitation process and provide more comfortability for patients. This paper focuses on implementing and construction of a three degree of freedom (DOF) (flexion/extension, adduction/abduction, and pronation/supination), low cost, lightweight, and portable robotic exoskeleton for wrist-forearm rehabilitation. SolidWorks software program and 3D printer technology are used to model and construct the proposed robotic exoskeleton structure. In addition, the anthropometric parameters of the normal human lower arm are considered for this exoskeleton to provide a range of motion (ROM) and velocity for the links, joints, which matches with the anatomical structure of human and also to avoid the excesses motions over the normal range. The exoskeleton is constructed by a 3D printer utilizing polylactic acid (PLA) plastic material. The proposed implementing structure of the robotic exoskeleton shows comfortable, lightweight, simple and economic as well.
\end{abstract}

Keywords: Exoskeleton, Robotic, Rehabilitation, 3D Printer.

$$
\begin{aligned}
& \text { الهيكل الخارجي الروبوتي: مدمج وممول ومُنفذ باستخدام تقنية طابعة ثلاثية الأبعاد }
\end{aligned}
$$

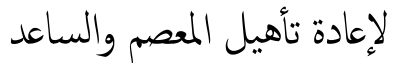

$$
\begin{aligned}
& \text { نور صبري شلال , وجدي صادق عبود } \\
& \text { الملاصة: } \\
& \text { تتطلب استعادة أنشطة الحياة اليومية بعد الإصابة بالسكتة الدماغية وإصابة الحبل الشوكي تمارين متكررة }
\end{aligned}
$$

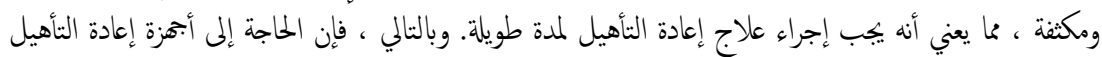

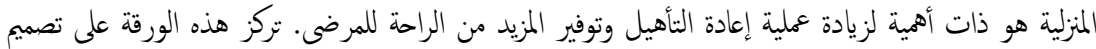

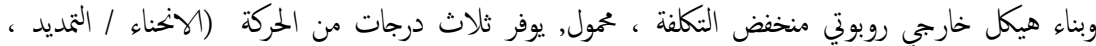

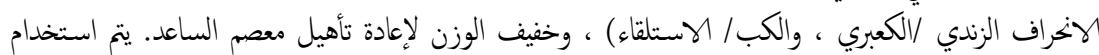

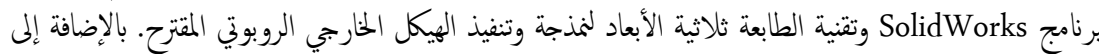

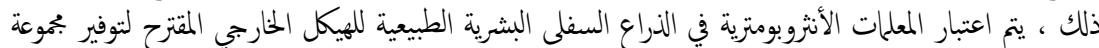

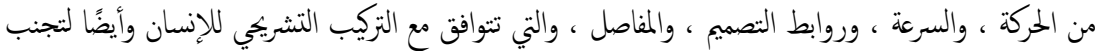

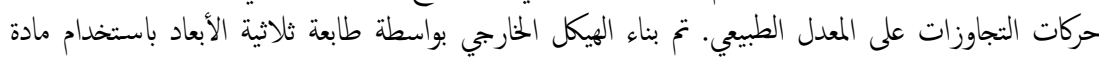

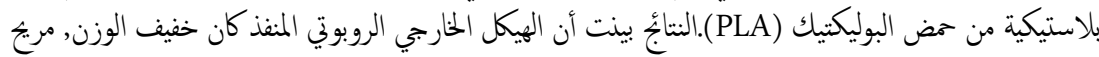

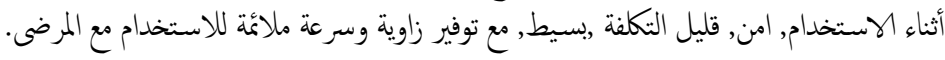

\section{Introduction}

Loss of the upper limb function (shoulder, elbow, wrist or hand or a combination of them) is in grow in elderly peoples, in addition, there are other conditions which are considered the most widespread causes of losing functions include stroke, spinal cord injury, cardiovascular disease, and occupational injuries. According to the World Health Organization, more than 15 million people are losses their daily activities due to the strokes and cardiovascular diseases [1]. Among these numbers, more than eighty percent of stroke survivors suffer an acute impairment of the arm and forty percent were either disables or chronically

NJES is an open access Journal with ISSN 2521-9154 and eISSN 2521-9162

This work is licensed under a Creative Commons Attribution-NonCommercial 4.0 International License 
impaired, thus leading to increase dependency of patients on their families and their society [2]. Since the number of patients is in increase, the therapists become more burdened and the number of them may decrease as compared to that of patients, in addition, the efficiency of the rehabilitation process decreases gradually and the patients become inactive. This will reflect in reducing the ability to restore the functional daily activities and independence. Introducing such rehabilitation devices are come to significantly solve the above problems, succeeding and improving the rehabilitation program. Several studies have shown that restoring and enhancing motor skills are related to intensive and repetitive functional tasks [3-8]. Although there is large development have been made on rehabilitation robotics, there are still limitations from the main goal, thus the robotic rehabilitation does not provide a proper function to regain bodily activities. These restrictions may result from the hardware system and control mechanism. The problems related to the hardware implementation represented in limitation in reflected range of motion, bulky and complexity, costly, uncomfortable, heavy in weight, incompatibility of the mechanical structure with that of the human, deficiency in safety purposes and power transmission methods [9-16]. In an appropriate form, the rehabilitation devices must be accessible for all patients through their existent in hospitals and rehabilitation centers. However, often the devices are existing in hospitals in their fixed states may be due to their bulky size or other reasons. These limitations offer a significant burden on patients in terms of their living away from hospitals and rehabilitation centers besides of its high cost.

As a result, many researchers have been interested in this field in which they developed and introduce new rehabilitation devices aiming to override the problems as much as possible [16-18]. However, the proposed devices still need several enhancements and reinforcements in terms of the technology usage in the implementation and manufacturing of them, the number of degree of freedom that shows by the considered device required to rehabilitate the defected limb to its optimum level, their restricted mode of operations which reflect on the efficiency of the rehabilitation process, their limitations to concerned on specific defected cases. Besides of possibility of making the devices bulky in size, heavy in weight and decreases the opportunity of home-based therapy.

This paper aims to exceed the limitations associated with the complexity of the mechanical implemented structure, heavyweight, enhance the device specifications represented by the number of degrees of freedom, mode of operations, and facility of the device of being compatible with all patients regardless of their age, gender, weight, and other parameters. The presented exoskeleton implemented such it offers three degrees of freedom (flexion/extension, adduction/abduction, and pronation/supination movements) for wrist and forearm rehabilitation. Such the device has been evaluated and examined in terms of its range of motion and velocities. The assessment of the device has been carried out lonely as well as with normal subjects.

The device was implemented with SolidWorks software program and manufactured using 3D printer technology as it shows the high accuracy of final construction with facilities of being deals and compatible with a wide range of materials such as the plastic one. The proposed exoskeleton has been constructed with polylactic acid (PLA) material. The choice of this construction material comes into its characteristics of being lightweight and cost less.

The next sections of this paper are arranged as follows: Section II explains the overall mechanical implementation and actuation system used, Section III presents the manufacturing process of the proposed exoskeleton, Section IV presents the electronic components used in addition to the control strategy, Section V clarifies the safety of the manufactured mechanical structure, Section VI details the results of the exoskeleton after analysis with and without subjects, finally, Section VII and VIII address the discussion and conclusion of this study respectively.

\section{The Mechanical Implementation and Actuation System \\ The proposed Considerations}

Since the rehabilitation devices are in direct contact with the human anatomical part, the structure of their parts must meet specific requirements. So it must be taken into account an extensive delicate consideration which involves the mechanical structure must be implemented in such a way that matches the human anatomical structure since the wrist and forearm joint provides movements around axis naturally not fixed, the availability of being adjusted for all patients regardless of age, gender, weight, and length which in turn the rehabilitation process can be accessible for all patients without any restrictions. The normal range of motions, the velocity of which the rehabilitative device must be work that deals with the case of a patient being under rehabilitation process, besides the safety and comfortability provided by the device which of the most important situations.

\section{Description of the Exoskeleton}

In association with the above considerations, the presented exoskeleton has been implemented in such a way meeting that considerations. The links and joint were implemented based on anthropometric data which are informed in table (1). The center of rotation on which the movements occur (at wrist joint) was achieved in such that is appropriate for all patients. It is considered the most important situation since all movements reflected by the wrist joint are occurring at that critical center, thus, any incompatible structure with the human center of rotation may make the rehabilitation process carried out with pain, inefficient, and ineffective therapy as a result. The links of its mechanical structure have been structured and connected with each other achieving the proposed three degrees of freedom with their normal range of motions and velocities. In addition, the mechanical structure has been carried out with the considerations of the patient's status during the operation's mode of the rehabilitation process. 
Table (1): Lengths of Lower Arm Segment [19].

\begin{tabular}{|l|l|l|}
\hline Segment & Males & Females \\
\hline Forearm & 15.70 & 16.00 \\
\hline Hand & 5.75 & 5.75 \\
\hline \multicolumn{2}{|l|}{ Segment lengths taken in (\%) of total body height. } \\
\hline
\end{tabular}

The process of implementing the exoskeleton was breaking down into two main steps. The first step includes the manufacturing of the forearm structure employed to provide a movement in one anatomical reference plane. The second step involves the manufacturing of the wrist structure which is further divided into two parts achieving the movements in the two anatomical reference planes. The subsequent sections detail the implementation process of each one of them.

All of these parts were implemented using the CAD software SolidWorks program. The choice of this program comes into the facilities of its simplicity, allowing the designer to make modifications and changing the implemented structure in a way that reduces the inaccuracies, solving the implementation problems, allowing the visualization of the overall structure including its range of motion before the final decision. In addition to the above facilities, it has a property of converting and storing the implemented mechanical structure in a form that compatible with every $3 \mathrm{D}$ printer type allowing the manufacturing of each piece then using 3D printer technology. Fig.1 shows the Anycubic mega-s 3d printer model which is used to produce the proposed exoskeleton in this study.

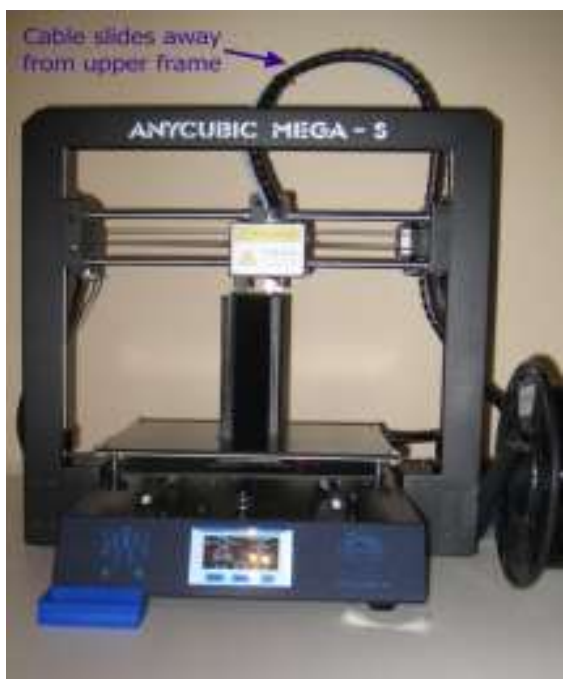

Figure (1) : 3D Printer Machine.

\section{Forearm Structure}

The mechanical structure of the forearm should be as easy as possible and provide an accurate and smooth motions facility, it is consists of several parts as shown in Fig. 2.

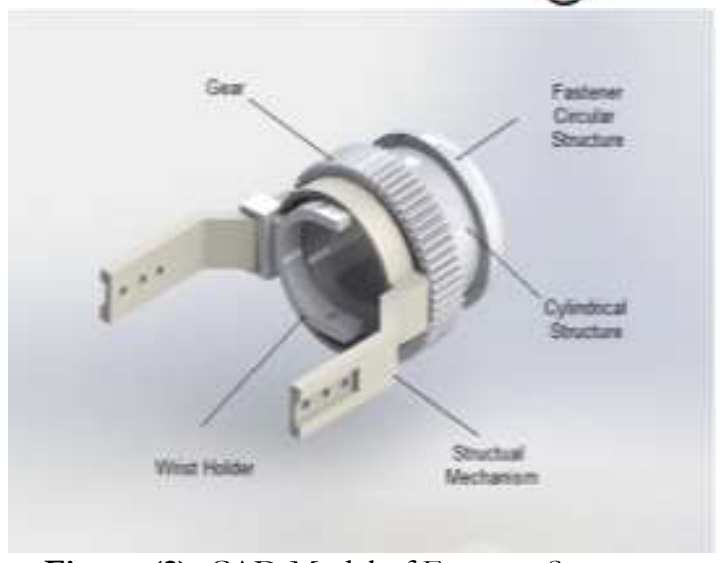

Figure (2): CAD Model of Forearm Structure.

The forearm part was implemented in a form that the distal end connected to the wrist part via screws at the contact point between the forearm and wrist parts. At the proximal end, it is connected to the base structure consequently. Its subdivisions include the cylinder, gears, wrist holder, fastener circular structure, and the structural mechanism. The cylinder structure exhibits the main function of the implemented forearm by allowing the movement of all parts of the robotic exoskeleton during the pronation/supination movement thus, achieving the rotation of both forearm and hand during the rehabilitation process. In addition, it is working as a carrying part of all forearm components and as a connection part between these components and the base structure. The gear components have been implemented to ensure the power transmission requirement (will be discussed in the actuation and power transmission mechanism section). The last part represents the structural mechanism extended from the cylinder to the wrist mechanism consisting of two links and a circle being build with one another to provide a rigid-complex frame, the wrist holder is carried out by this mechanism. Another part of the forearm mechanism has been implemented which is a circular structure of one end being attach and enter the cylinder structure while the other end has a likely curved form to prevent the risk for the subject when entering his/her lower arm, the function of this circular structure was act as a fastener for the cylindrical structure preventing it from step out during the operation of the exoskeleton. The forearm structure represents the pronation/supination part of the exoskeleton provides the same range of motion as that of the normal human one.

\section{Wrist Structure}

The wrist structure represents the most critical part that must be taken into consideration during the implementation process as it contains the axis of rotation that must match that of the human one. Along with it, the two motion occur including the flexion/extension and adduction/abduction movements. The wrist mechanism consists of two main parts each of them is configured to provide the two above proposed movements take place through the axis of rotation.

\section{Flexion /Extension Part}

Fig.3 shows the flexion/extension part which is responsible for providing the flexion/extension movement occurring in the sagittal plane. This part 
was implemented to match two ends representing the proximal end which connects to the forearm mechanism, and the distal end that connects to the second part of the wrist mechanism (adduction/abduction part) which in turn permitting a connection to the forearm mechanism, thus, these parts will rotate during pronation/supination movement allowing the rotation of the wrist mechanism and reflects this movement to the subject wearing it.

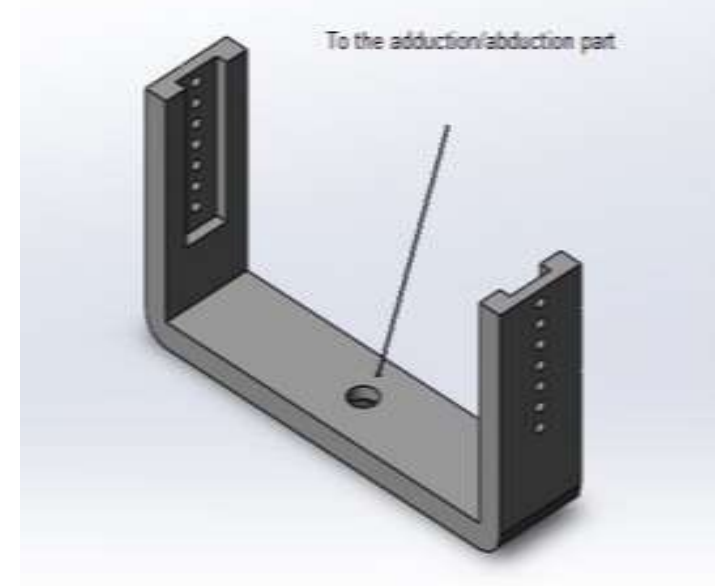

Figure (3): CAD Model of Wrist Flexion / Extension Part.

\section{Adduction/Abduction Part}

The implemented part is shown in Fig. (4) provides the adduction/abduction movement of the exoskeleton in the transverse plane, it was implemented that attaching to the flexion/extension at the axis of rotation. This part is implemented such that it consists of three parts connecting with each other, the first part connecting to the flexion/extension part, while the other part is connecting to handle part which facilities the movement of the last one during adduction/abduction motion. The first two parts are also connected to each other through springs thus, provide smooth and more flexible movement, also the structure being less rigid and more resilient.

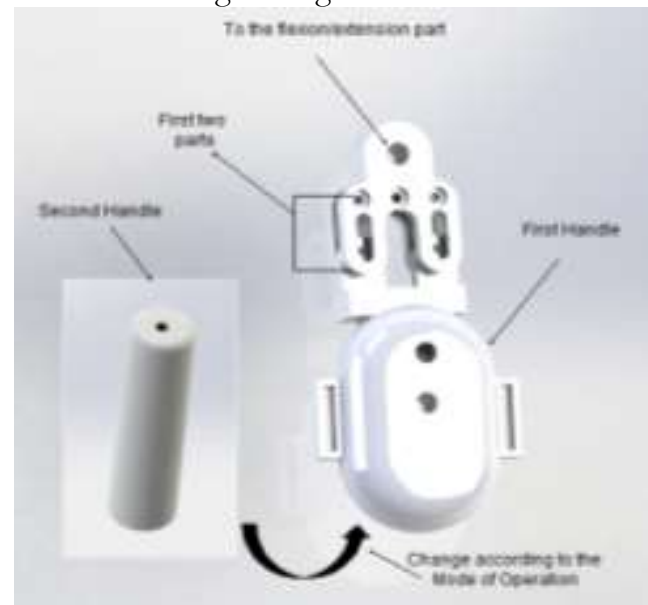

Figure (4): CAD Model of Adduction / Abduction Part.
The handle mechanism represents the end rest structure. It carries the function of supporting the subject's hand during the overall motions of the robotic exoskeleton. Two-handle mechanisms (see Fig. (4)) have been implemented according to the patient's requirements, each mechanism has its own function, and its utilization time being used according to the rehabilitation progress.

The first handle was implemented to be used during the first stage of the rehabilitation process (during the passive mode of operation) when the patients aren't able to move their hands. It has been noted that the stroke, spinal cord injury, and wrist drop patients were not able to hold with their hands, thus, the handle structure must allow them to rest their hand at their current state without the need to form any movement to be compatible with the structure. According to this property, the handle mechanism structured to allow them only to rest their hand on it, and the process of treatment takes its course. It fortified with a strip passed through its sides ends holding the hand. It is implemented in a way that meets the requirements of comfortability, compatibility for the human hand. The second mechanism was implemented to be used through the progressed stage of rehabilitation process (during the active mode of operation) that required the patient to flexes its hand to be compatible with the implemented mechanism i.e., when the patients have the ability to move their hand and they exhibit some activities in their muscles.

Moreover, both the structural mechanism of the forearm structure and the flexion/extension part of the wrist structure (See Figs. (2) and (3)) were reinforced with holes exist on the sides of their distal ends functions to permit the exoskeleton to be adjusted according to the patient's rotational axis by elevating / lowering, progressing/delaying these parts respectively.

\section{Implementation of the Accessories}

In addition to the above main components of the exoskeleton, there are other mechanisms constructed the exoskeleton functions to provide several destinations such as supporting, resting, and balancing both the subject and the robotic exoskeleton. These parts include the wrist holder and base structure as shown in Fig. 5. The base structure is responsible for the overall supporting and balancing of the exoskeleton while its motions, in addition to its function for human lower arm resting structure, it also configured by making a place for holding the motor allowing it for the attachment with the actuation and power transmission mechanisms. The wrist holder is manufactured as a slide structural mechanism permitting to applied it for all patients according to their restricted requirements, it is configured to hold the wrist attaching with the tape to ensure the supports of the subject, both of above structures have been reinforced with a cushioned materials which attached to the inner frame of them of which the subject's forearm placed to provide the comfortability during the rehabilitation process. 


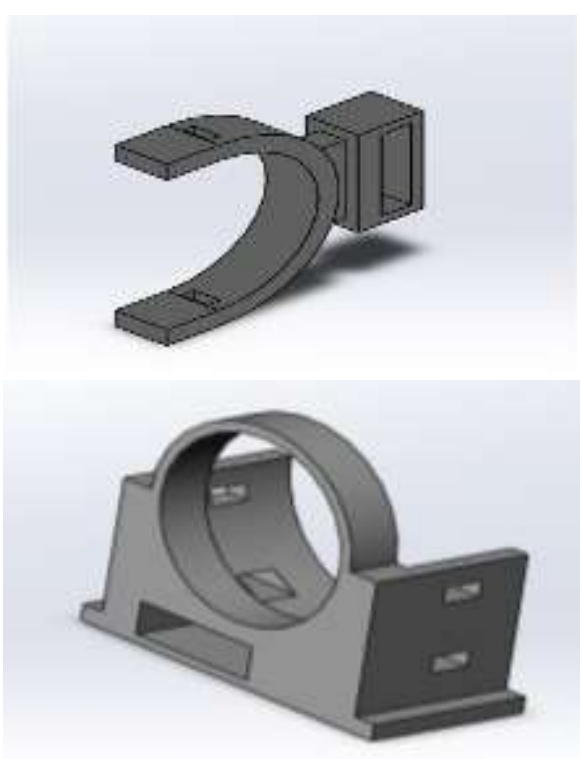

Figure (5): CAD Model of above: Wrist Holder, below: Base.

Final Parts and Assembly of the Exoskeleton

The exoskeleton subdivisions parts are assembled in SolidWorks software program to construct the final shape of the proposed exoskeleton structure and to be ready for printing in the $3 \mathrm{D}$ printer which utilizing the PLA plastic material as shown in Fig. 6.

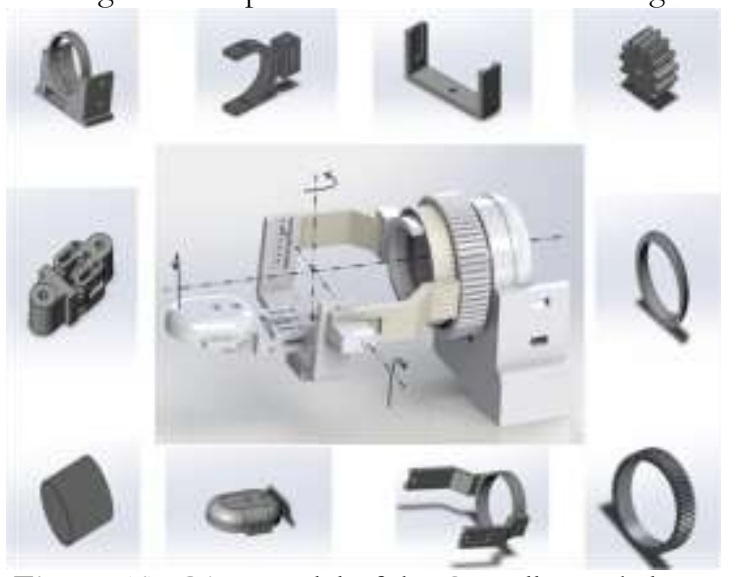

Figure (6): CAD Model of the Overall Exoskeleton.

\section{Actuator Selection}

The actuators must be chosen carefully since they determine the efficiency and quality of the device. In this study, the DC servo motors chose according to the requirements criteria involve the torque, range of motion, weight, and size of it. The torque requirement has been selected based on normal human daily activities [23]. Added to this a safety factor, patient weight, and motor inertia. Three DC servo motors have been chosen to achieve the proposed three degrees of freedom of the presented exoskeleton. Such motors include the Tower Pro mg995 servo motor that used to actuate the adduction/abduction part while the flexion/extension part and forearm part was actuated using the two DS3218 Digital servo motors hierarchy. The overall exoskeleton also rotated as a result of the movement of the forearm part which substantially achieving the pronation/supination movement of the lower arm.

\section{Actuation Mechanism}

During the actuation mechanism, the rotational motions of the first two servo motors are translated to the flexion/extension and adduction/abduction parts via the attaching screws. These rotational movements resulted in rotation of these parts within the proposed range of motions thus, achieved the flexion/extension and adduction/abduction movements of the exoskeleton that reflected to the human hand. The forearm mechanism achieved its movement through rotation of the DS3218 Digital servo motor being translated to a mechanism consisting of two gears (see Fig. 7). The translation of this movement occurs in a strategy that the motor attached to a small gear (bevel gear implemented with 16 teeth) therefore it is known as driver gear, this gear mates with a driven gear larger than the driver one which in turn connected to the cylindrical structure of the forearm exoskeleton part as shown in Fig. (7). With this mechanism, the rotational movement is translated to the spur gear allowing it to rotate the whole exoskeleton to achieve the pronation/supination movement of the human's lower arm.

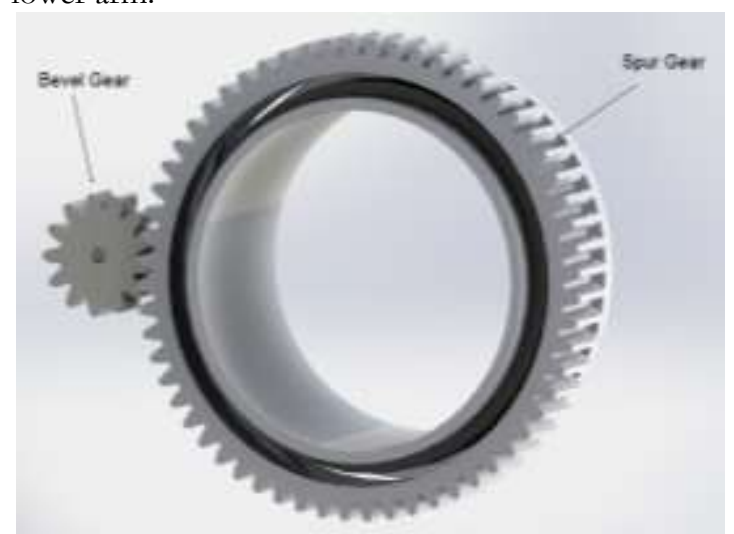

Figure (7): Gear Power Transmission.

\section{Exoskeleton Manufacturing Process}

The manufacturing process of the exoskeleton started with choosing a suitable material then constructed off the exoskeleton parts. All parts constructed the exoskeleton was built and configured by an additive technique which is a $3 \mathrm{D}$ printing, it is a method of constructing approximately any form of the computer-aided design (CAD) by utilizing thermoplastic materials such as PLA, acrylonitrile butadiene styrene (ABS) and polyethylene terephthalate (PET). It is characterized by its naivety and customization, in addition to its low-cost manufacturing. In order to print any implemented part, the only requirements to do by the user are entering the desired thermoplastic filament and the required file to be printed.

The proposed exoskeleton was printed using PLA thermoplastic material, selection of this material has been decided with the considerations of its low cost, weight of the components that placed with the exoskeleton structure (such as motors), the weight of the lower arm (based on the anthropometric data presented in table (2)) and the surrounding environment of which the exoskeleton contributes, include the thermal, tensile and chemical 
environment. Because the exoskeleton doesn't expose to large external forces, and being in an environment may be exposed to a slight change in temperature and chemical properties, it is founded that the PLA material appropriate to withstand the above environmental properties, in addition to meeting the requirements of being low in cost and lightweight $[20,21]$. Table (3) shows the properties of the PLA material.

Table (2): Weights of Lower Arm Segment [19].

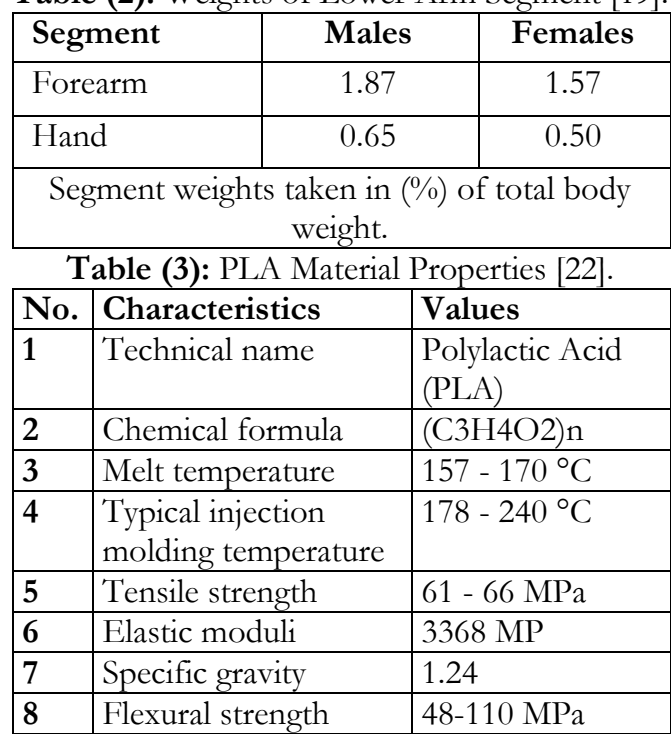

By using the Fused deposition molding technology (FDM), the exoskeleton has been made, it is operated as furnishing it with a filament or metal wire from a coil that supplying to an extrusion nozzle, it can shift in a horizontal and vertical direction navigable by software of computer-aided manufacturing (CAM), each subdivision of the exoskeleton was created by extruded the material that melted with this pre-heated nozzle. One of the features that must be noted is that the parts being printed have significant strength along the plane of the printing in comparison with that normal to the plane, throughout the printing execution, each heating layer comes in contact with the sequent layer and drawn with each other. As the next layer is printed, the previous one is cooling down and consolidate [24].

\section{Forearm Parts and their Assembly}

The printing technique promises a significant accuracy when it comes to dimension; i.e. there was not much difference between the final shape dimensions and the cad model. The forearm parts are connected together to form the final construction of the forearm structure (see Fig.8), each part was constructed in a way that doesn't need screws or pins, thus making the structure easier.

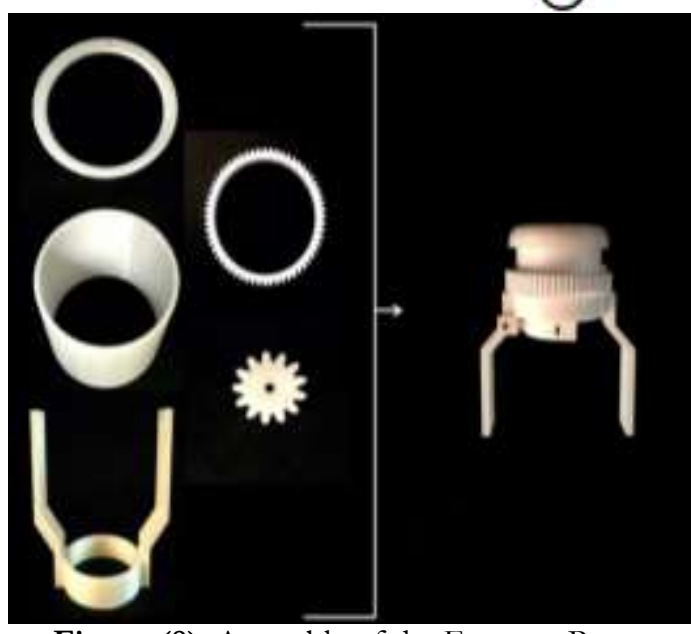

Figure (8): Assembly of the Forearm Parts.

Wrist Parts and their Assembly

The wrist structure was printed as two parts, the flexion/extension part, and the adduction/abduction part, the adduction/abduction part was printed as three subdivisions. Fig. (9) shows the wrist subdivisions and its assembly.
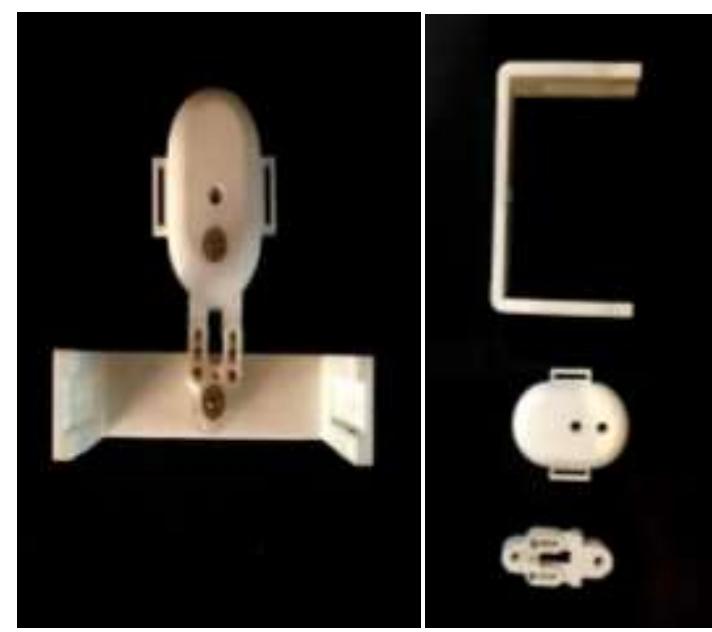

Figure (9): Assembly of the Wrist Parts.

The wrist part was connected to the forearm part to form the whole exoskeleton. Fig. 10 shows the assembly of the forearm-wrist structure.

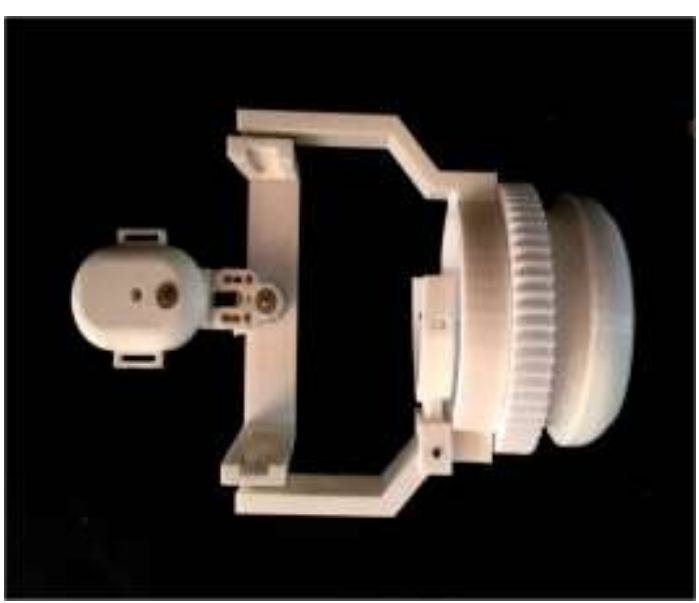

Figure (10): Forearm-Wrist Parts Assembly.

The forearm-wrist structure was connected with the base structures. An additional supporter base was implemented to lift off the overall exoskeleton to perform the reflected movements for each link at its 
optimum state and to achieve the proposed range of motion without making any undesired contact with other structures, in other meaning, allowing the exoskeleton to move freely. Subsequent figures (1214) detail the steps of the exoskeleton assembly with the the servomotors positioned and base structure with it.

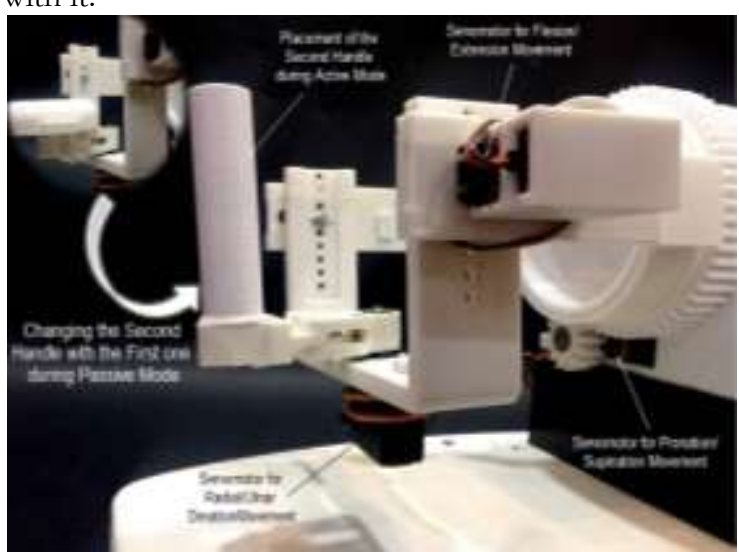

Figure (11): Placement of Servomotors.

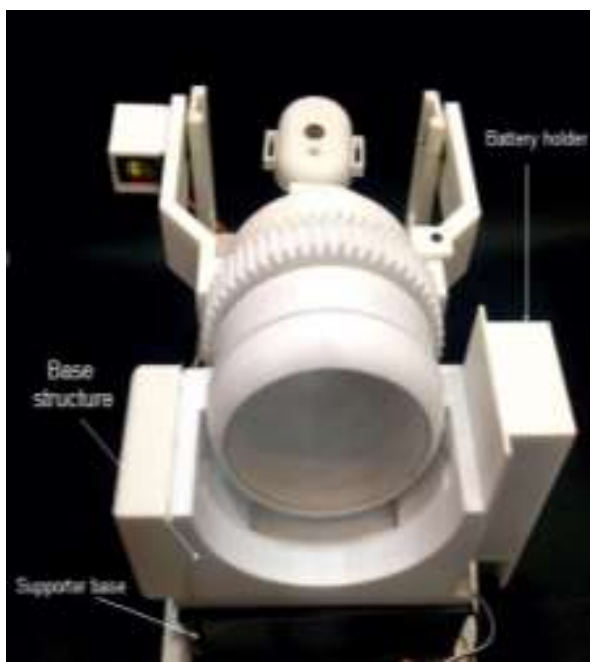

Figure (13): Support the Overall Exoskeleton with Bases Structure.

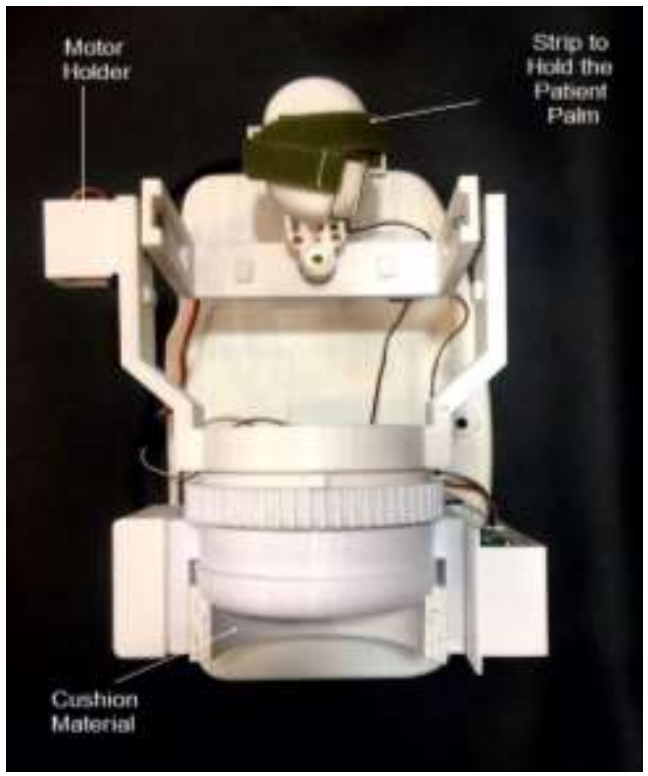

Figure (14): Final Assembly of the Exoskeleton.

\section{Safety}

The most important situation leading to mechanical manfacturing structure a rehabilitative exoskeleton is to ensure the safety of it which is represented by limiting the range of motions that the exoskeleton reflects to prevent it from exceeding that of normal one, in addition to equipped with motors that can be programmed in a way to limit the exoskeleton motion to specified angles and velocities as well (see table(4)). Each part of its mechanical structure must have no sharp edges or scratches that may result in undesirable or pain to the patient wearing it which in turn make the rehabilitation process unaffected consequently. In this study, the mechanical structure has been carried out with the mechanical stoppers (see Fig. 15) placed in specific locations in wrist and forearm mechanisms to restrict the range of motion to that of a normal human.

Table (4): Normal Human Average Data.

\begin{tabular}{|c|c|c|c|}
\hline Movement & \multicolumn{2}{|c|}{$\begin{array}{l}\text { Range of } \\
\text { Motion }\left(\mathrm{deg}^{\circ}\right)\end{array}$} & $\begin{array}{l}\text { Velocity } \\
\left(\text { deg}^{\circ} / \mathrm{s}\right)\end{array}$ \\
\hline Flexion & \multicolumn{2}{|c|}{$75^{\circ}$} & 1970.168 \\
\hline Extension & \multicolumn{2}{|l|}{$70^{\circ}$} & 2175.768 \\
\hline Adduction & \multicolumn{2}{|l|}{$25^{\circ}$} & 27496.58 \\
\hline Abduction & \multicolumn{2}{|l|}{$35^{\circ}$} & 22955.46 \\
\hline Pronation & $80^{\circ}$ & \multirow[t]{2}{*}{$150^{\circ}$} & \multirow[t]{2}{*}{803.548} \\
\hline Supination & $70^{\circ}$ & & \\
\hline
\end{tabular}

Besides, the exoskeleton was made based on these limitations to avoid obstacles that could affect the rehabilitation process. Also, it was reinforced with a cushioned material to increase the comfortability for the patient thereby making the rehabilitation process more effective.
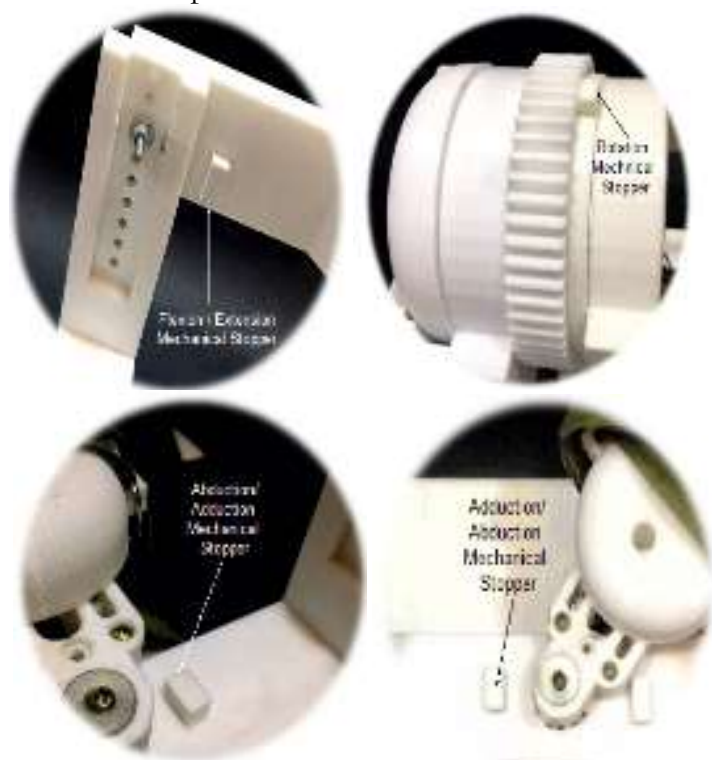

Figure (15): Mechanical Stoppers.

\section{Control System}

In order to achieve the performance of the device in terms of its reflected range of motions and velocity data besides its ability to overcome the carried load, it was already equipped with some electronic components including the gyroscope sensor for angle measurement which is placed at the distal end of the exoskeleton (in order to pick up all three 
movements of the exoskeleton), a microcontroller presented in an Arduino mega as a controller through it the motors and gyroscope sensor were programmed to provide the range of motion, velocities information emulating that of normal human and to evaluate this information respectively. The control strategy of the exoskeleton's motors are based on the angles information forwarded by the gyroscope sensor, in a a way that when there are no angles data exhibits by the patient, the exoskeleton will operate in a passive mode of operation, otherwise, it will enter the active mode of operation consequently.

\section{Results}

The exoskeleton device has been evaluated for its range of motion and velocities alone on one hand and with normal subjects on the other hand. It was also tested for its safety, comfortability, its endurance to carry and withstand high loads, its availability for different patients regardless of age; length; and gender. These testing procedures have been done to ensure that the exoskeleton is valid to be applied with patients to rehabilitate them for regaining their activities of daily living. Table (5) shows the resulting range of motions and velocity information for the exoskeleton.

Table (5): Range of Motions and Velocities Information of the Exoskeleton.

\begin{tabular}{|l|l|l|}
\hline $\begin{array}{l}\text { Type of } \\
\text { motion }\end{array}$ & $\begin{array}{l}\text { Exoskeleton } \\
\text { angle }(\mathbf{d e g})\end{array}$ & $\begin{array}{l}\text { Exoskeleton } \\
\text { Velocity }(\mathbf{d e g} / \mathbf{s})\end{array}$ \\
\hline Flexion & $70^{\circ}$ & 1332.645 \\
\hline Extension & $75^{\circ}$ & 1528.402 \\
\hline Adduction & $25^{\circ}$ & 1908.026 \\
\hline Abduction & $35^{\circ}$ & 1826.868 \\
\hline Rotation & $150^{\circ}$ & 493.468 \\
\hline
\end{tabular}

Figures (16-21) show the movements of the exoskeleton in addition to their corresponding curves denoting the reflected range of motions and velocities respectively.

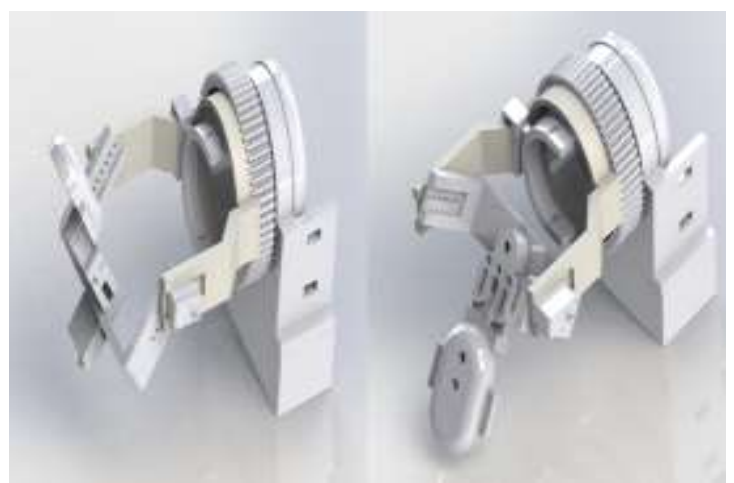

Figure (16): Extension (left) and Flexion (right) Movement of the Exoskeleton.
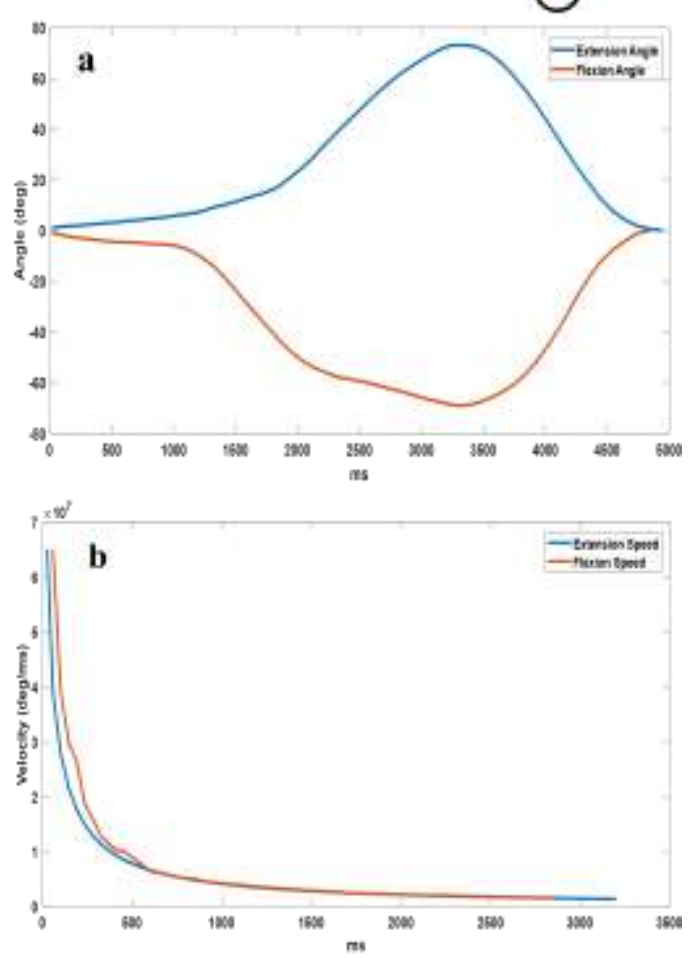

Figure (17): ROM (a) and Velocity (b)

Flexion/Extension Analysis of the Exoskeleton.

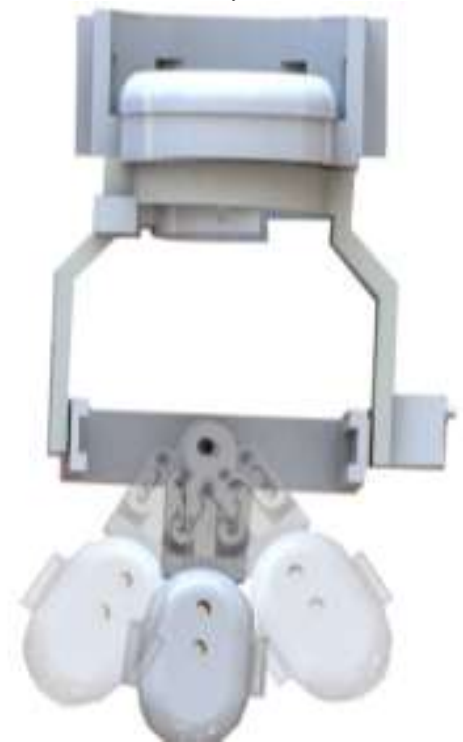

Figure (18): Adduction / Abduction Movement of the Exoskeleton.

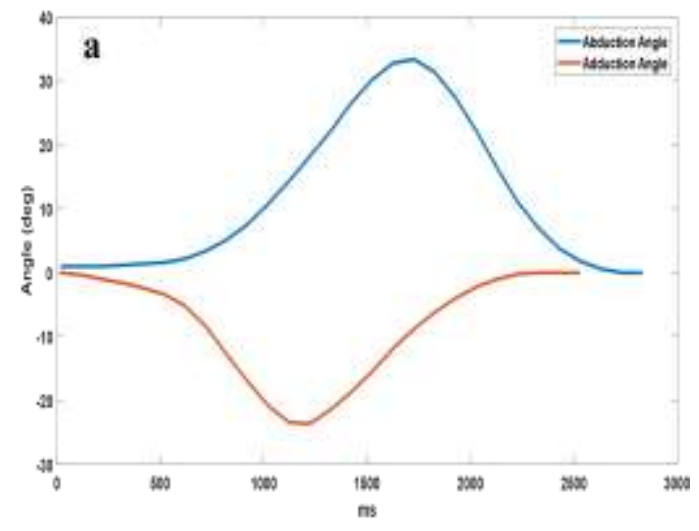




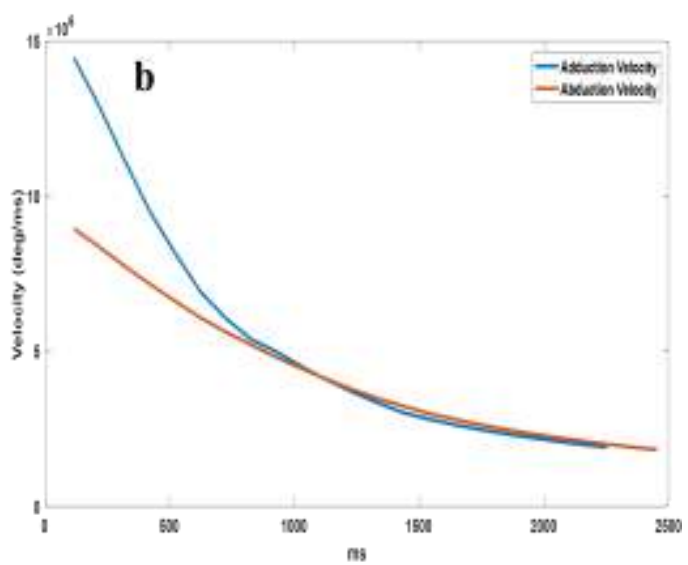

Figure (19): ROM (a) and Velocity (b) Analysis of the Adduction/Abduction Movement of the Exoskeleton.

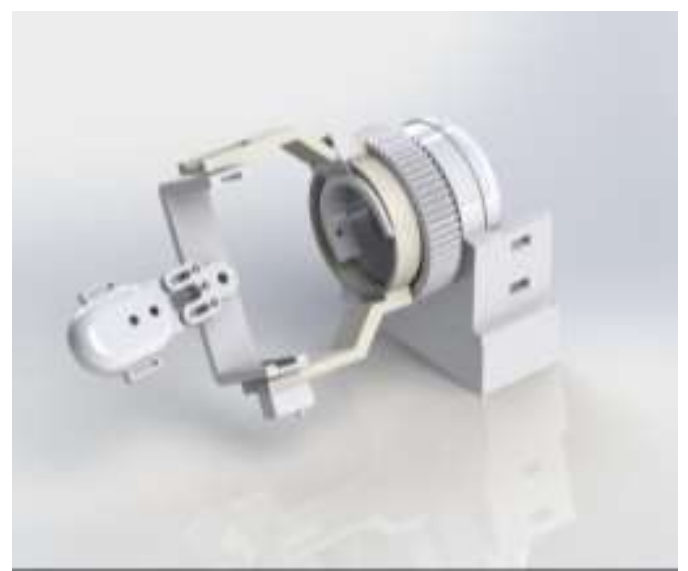

Figure (20): Rotational Movement of the Exoskeleton.
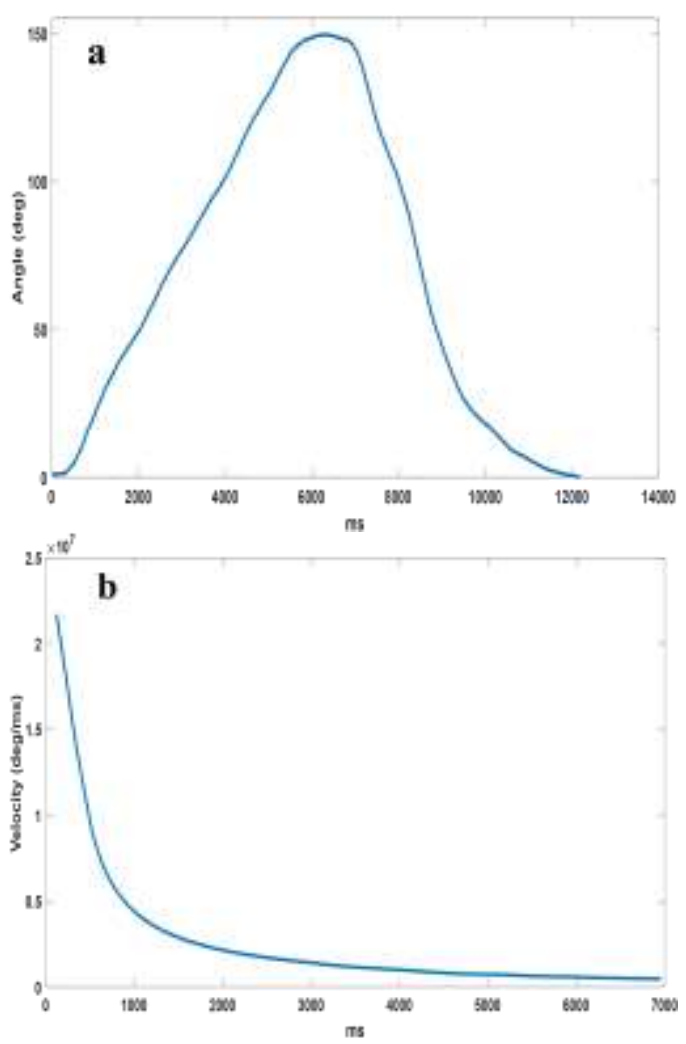

Figure (21): ROM (a) and Velocity (b) Analysis of the Rotational Movement of the Exoskeleton.
Figures. (22-25) show the exoskeleton application with a normal subject, testing results of the reflected ranges of motion exhibited by the exoskeleton for the normal subject as wearing it subsequently.

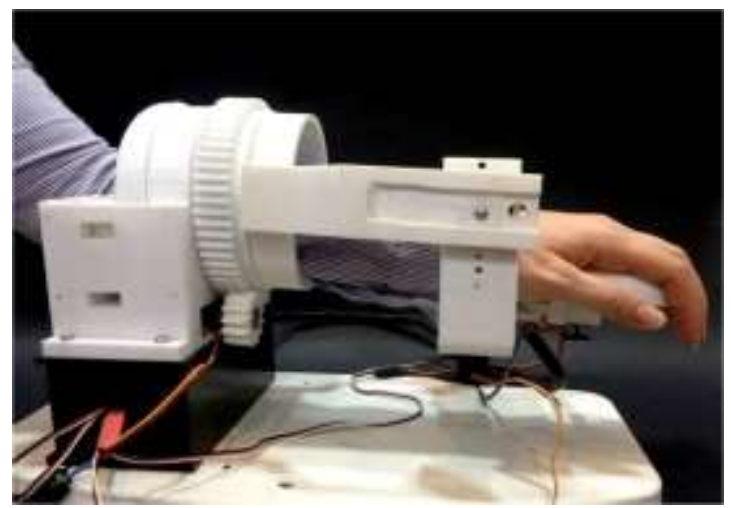

Figure (22): Exoskeleton Applied with Normal Subject.

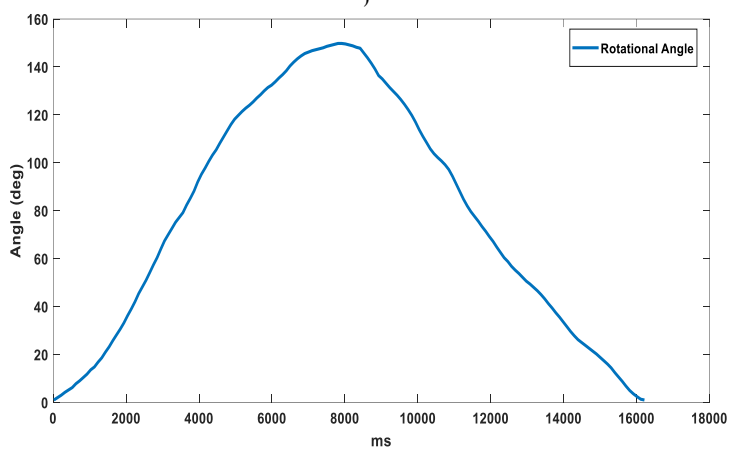

Figure (23): Rotational Angle of Normal Subject while Wearing the Exoskeleton.

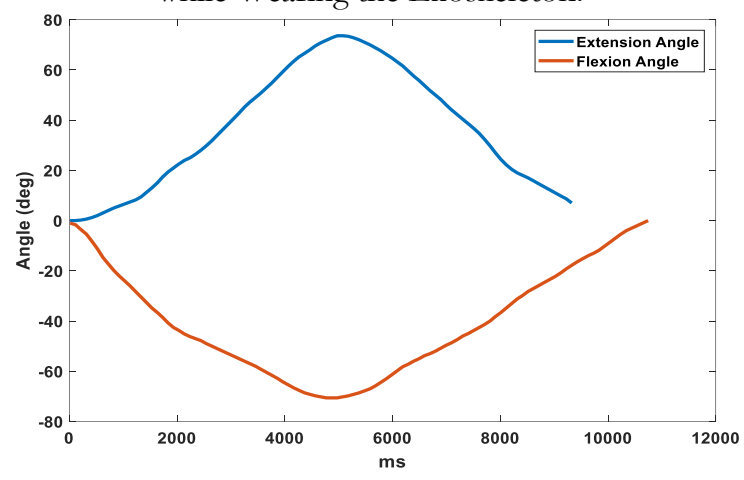

Figure (24): Flexion/Extension Angle of Normal Subject while Wearing Exoskeleton.

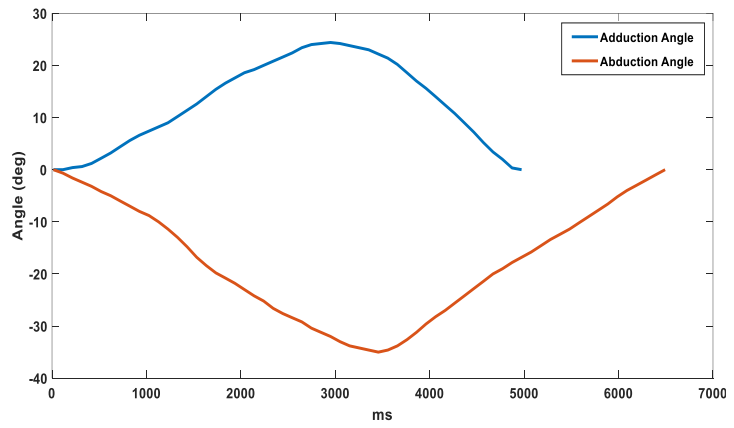

Figure (25): Adduction/Abduction Angle of Normal Subject while Wearing the Exoskeleton. 


\section{Discussion}

This study goals to implement and construct a novel exoskeleton exceeding the limitations and obstacles found in other proposed exoskeleton structures. The manufacturing process was treated with lightweight, costless, withstand material such making the device carried with specific features in terms of its portability, low in cost, used among several patients regardless of their weight. This device was implemented to exhibit three degree of freedom to provide a complete qualification of wrist and forearm joints through the presetting training program with two mode of operation that decided according to the patient's rehabilitation progressing and his type of defect. The exoskeleton shows the reflected range of motions as that of normal human with velocity information slightly little than the half of normal human because the exoskeleton was programmed to work primarily with lower velocities, then the proposed velocities will be increased as the patient enhanced with the rehabilitation process.

\section{Conclusion}

Impairment of the motor function of the upper limb due to defected conditions such as SCI and stroke is of utmost problem due to their effectiveness of inhibiting the activities of daily living, an intensive rehabilitation program can facilitate the way for patients to restore their daily functions. This rehabilitation program can be done with the therapists, but there are several limitations associated with this strategy involving the effectiveness of the rehabilitation process with the therapists, the level of the therapists' knowledge, and the number of patients as compared with that of therapists in hospitals and rehabilitation centers. This study mostly concerned on mechanical implementation structure and its construction as well. From the mechanical point of view, the overall mechanical structure offers links and joint matching that of the human one, in addition to its facilities of the presented rotational axis that emulating the center of rotation of the wrist joint. Moreover, this axis can be adjusted according to that of the patient's axis. These features of its implementation increase the opportunity of it to be available for different patients of different anthropometric parameters. Besides of the overall little maintenance requirements. The device also presents two-mode operation, each of them requires a change in its handle structure according to the patient status which no study has shown such structure that may limit the rehabilitation therapy associated with their devices. The testing results of the device alone and with a normal subject show excellent performance, reflects a smooth movement, and achieve a range of motions and velocities as that of the normal human. These overall features making the device promises an effective, complete, and support the home-based rehabilitation therapy as it implemented with compact size such making it portable and facilitate its transition. Besides, the overall device being low in cost as it implemented from low cost material thus, increases the availability for wide ranges of patients, making the rehabilitation process costless and increase the repetitions of therapy as well. As a result, it is ready to be applied with SCI and stroke patients which in turn represents the role of the physiotherapist.

\section{Acknowledgment}

I would like to put a great thank for Assist prof. Dr. Auns Q Al-Neami for her help and advices to complete this study.

\section{References}

[1] J. Mackay and G. Mensah, Atlas of Heart Disease and Stroke (Nonserial Publication, World Health Organization, Brighton, UK,), 2004.

[2] V. M. Parker, D. T. Wade and H. R. Langton, "Loss of arm function after stroke: Measurement, frequency, and recovery," Int. Rehabil. Med. 8(2), 69731986.

[3] C. J. Winstein, A. S. Merians and K. J. Sullivan, "Motor learning after unilateral brain damage," Neuropsychologia 37(8), 975-987, 1999.

[4] G. N. Lewis and J. A. Rosie, "Virtual reality games for movement rehabilitation in neurological conditions: How do we meet the needs and expectations of the users?" Disabil. Rehabil. 34(22), 1880-1886, 2012.

[5] D. A. Mauro, "Virtual Reality-Based Rehabilitation and Game Technology," 1st International Workshop on Engineering Interactive Computing Systems for Medicine and Health Care (EICS4Med) Pisa, Italy , pp. 48-52,2006.

[6] C. Carignan, J. Tang and S. Roderick, "Development of an Exoskeleton Haptic Interface for Virtual Task Training," 2009 IEEE/RSJ International Conference on Intelligent Robots and Systems (IROS 2009), St. Louis,pp. 3697-3702,2009.

[7] M. Cameirao, S. Badia, E. Oller and P. Verschure, "Neurorehabilitation using the virtual reality based rehabilitation gaming system: Methodology, design, psychometrics, usability and validation," J. NeuroEng. Rehabil. 7(1), 48,2010.

[8] P. C. Stroke," Robotic devices and brain-machine interfaces for hand rehabilitation post-stroke", $J$ Rehabil Med, 49, 449-460, 2017.

[9] P. Garrec, J. P. Friconneau, Y. Measson, \& Y. Perrot," ABLE, an innovative transparent exoskeleton for the upper-limb," In 2008 IEEE/RSJ International Conference on Intelligent Robots and Systems (pp. 1483-1488).

[10]A. Frisoli, F. Salsedo, M. Bergamasco, B. Rossi , \& M. C. Carboncini, "A force-feedback exoskeleton for upper-limb rehabilitation in virtual reality. Applied Bionics and Biomechanics, 6(2), 115-126, 2009.

[11] C. D. Takahashi, L. Der-Yeghiaian, V. Le , R. R. Motiwala, \& S. C. Cramer, "Robot-based hand motor therapy after stroke. Brain", 131(2), 425-437,2008.

[12] R. A. R. C. Gopura, K. Kiguchi and L. Yang, "SUEFUL-7: A 7-DOF Upper-Limb Exoskeleton Robot with Muscle-Model-Oriented EMG-Based Control," 2009 IEEE/RSJ International Conference on Intelligent, Robots and Systems (IROS 2009), Piscataway, NJ (Oct. 11-15, 2009) pp. 1126-1131,2009. 
[13] K. Homma and T. Arai, "Design of an Upper Limb Motion Assist System with Parallel Mechanism,"

Proceedings of the 1995 IEEE International Conference on Robotics and Automation. Part 1 (of 3), Nagoya, Japan (May 21-27, 1995) pp. 1302-1307,1995.

[14] N. G. Tsagarakis and D. G. Caldwell, "Development and control of a 'soft-actuated' exoskeleton for use in physiotherapy and training," Auton. Robot. 15(1), 21-33, 2003.

[15] C. Carignan, J. Tang and S. Roderick, "Development of an Exoskeleton Haptic Interface for Virtual Task Training," 2009 IEEE/RSJ International Conference on Intelligent Robots and Systems (IROS 2009), St. Louis, MO pp. 3697-3702,2009.

[16] A. Hassanin, D. Steve, and N. Samia, "A novel, soft, bending actuator for use in power assist and rehabilitation exoskeletons" , IEEE/RSJ International Conference on Intelligent Robots and Systems (IROS), pp. 533-538,2017.

[17] C. Lambelet, M. Lyu , D. Woolley, R. Gassert, \& N. Wenderoth, "The eWrist - a wearable wrist exoskeleton with sEMG-based force control for stroke rehabilitation", In 2017 International Conference on Rebabilitation Robotics (ICORR) (pp. 726-733).

[18] I. Yamamoto, M. Matsui, T. Higashi, N. Iso, K. Hachisuka, \& A. Hachisuka," Wrist rehabilitation robot system and its effectiveness for patients", Sensors and Materials, 30(8), 1825-1830, 2018.
[19] Hall S., "Basic biomechanics," McGraw-Hill, Sixth Edition, 2012.

[20] P. R. Culmer, A. E. Jackson, S. Makower, R. Richardson, J. A. Cozens, M. C. Levesley, \& B. B. Bhakta," A control strategy for upper limb robotic rehabilitation with a dual robot system", IEEE/ASME Transactions on Mechatronics, 15(4), 575-585, 2009.

[21] N. S. Korivi, "Preparation, characterization, and applications of poly (ethylene terephthalate) nanocomposites", In Manufacturing of Nanocomposites with Engineering Plastics, Woodhead Publishing, pp. 167-198, 2015.

[22] M. King, B. Phillips, M. Shively, V. Raman, A. Fleishman, S. Ritter, \& K. Mehta, "Optimization of prosthetic hand manufacturing", In 2015 IEEE Global Humanitarian Technology Conference (GHTC) (pp. 59-65).

[23] J. H. Beekhuis, A. J. Westerveld, H. van der Kooij, \& A. H. Stienen," Design of a self-aligning 3DOF actuated exoskeleton for diagnosis and training of wrist and forearm after stroke", In 2013 IEEE 13th International Conference on Rehabilitation Robotics (ICORR) (pp. 1-5).

[24] V. Adhikari, " Design of A Novel Task-Based Knee Rehabilitation Exoskeleton Device With Assist-As-Needed Control Strategy", Thesis, Uttar Pradesh Technical University, 2017. 